\title{
COMPUTER-ASSISTED DRUG DESIGNING OF TRIAZOLE DERIVATIVE OF NOSCAPINE AS TUBULIN-BINDING ANTICANCER DRUG
}

\section{PUJA KUMARI ${ }^{1 \#}$, VINEETA DIXIT ${ }^{2 \#}$, ATUL KUMAR TIWARI ${ }^{2}$, SAURABH SAXENA ${ }^{3}$, NAVEEN KUMAR VISHVAKARMA ${ }^{1}$, PRADEEP KUMAR NAIK ${ }^{4}$, DHANANJAY SHUKLA ${ }^{1 *}$}

${ }^{1}$ Department of Biotechnology, Guru Ghasidas Vishwavidyalaya, Bilaspur - 495009 , Chhattisgarh, India. ${ }^{2}$ Department of Botany, Guru Ghasidas University, Bilaspur - 495 009, Chhattisgarh, India. ${ }^{3}$ Government Science College, Ambikapur, Chhattisgarh, India, Department of Medical Laboratory Sciences, Lovely Professional University, Punjab, India. ${ }^{4}$ Department of Life Science, Sambalpur University, Sambalpur - 768 019, Odisha, India. Email: sdhannu@gmail.com

\#Both authors contributed equally to this work.

Received: 05 April 2018, Revised and Accepted: 11 July 2018

\begin{abstract}
Objective: Microtubule-interfering drugs are commonly used to treat malignant disorders owing to indispensable role of this cytoskeletal element. These drugs include paclitaxel, docetaxel, and the Vinca alkaloids; however, owing to their non-selective action and overpolymerizing effects, these chemotherapy drugs are confounded by complications with serious toxicity (particularly, peripheral neuropathies, gastrointestinal toxicity, myelosuppression, and immunosuppression) (by taxanes) or depolymerizing effects (by Vincas) on microtubules. Thus, there is urgent need to explore novel tubulin-binding agents that are significantly effective and comparatively less toxic compared to currently available drugs for the treatment of human cancer. The current study focuses fusion of two novel anticancer compounds with low toxicity, i.e., noscapine and triazole to generate a new ligand derivative.
\end{abstract}

Methods: Using computer-aided drug designing approach and molecular docking, molecular interaction of these derivatives with $\alpha \beta$-tubulin heterodimer was confirmed and investigated by molecular docking along with dynamics simulation.

Results: A greater affinity of the newly designed ligands for binding to tubulin was predicted. The predictive binding free energy ( $G_{\text {bind,pred }}$ ) of these derivatives (ranging from -10.5178 to $-16.8473 \mathrm{kcal} / \mathrm{mol}$ ) based on linear interaction energy method with a surface generalized born continuum salvation model showed improved binding affinity with tubulin as compared to the lead compound. natural $\alpha-N o s c a p i n e ~(-5.505 \mathrm{kcal} / \mathrm{mol})$. The binding energy of ligand determined using LigX, i.e., MM/GBVI was found to be $-23.208 \mathrm{kcal} / \mathrm{mol}$.

Conclusion: We found that designed derivative compounds have better efficacy as compared noscapine and triazole.

Keywords: Noscapine, Triazole, Tubulin heterodimer, Molecular docking, Surface generalized born.

(C) 2018 The Authors. Published by Innovare Academic Sciences Pvt Ltd. This is an open access article under the CC BY license (http://creativecommons. org/licenses/by/4. 0/) DOI: http://dx.doi.org/10.22159/ajpcr.2018.v11s2.28586

\section{INTRODUCTION}

Over the years, various investigations come up with novel therapeutic interventions to prevent and/or treat neoplastic disorder and associated concerns such as metastasis. Metastasis, spreading of cancer lesions from primary site of carcinogenesis to secondary sites, requires cellular phenotype transition (epithelial-mesenchymal transition) which involves reprogramming cytoskeletal elements [1]. Research evidences also report that early stages of malignant transformation of neoplastic cells essentially involve alteration of cytoskeletal structure of cells [2]. A good proportion of chemotherapeutic agents act through interfering microtubule function in transformed cells. Among these, tubulin-binding drugs are most successful and constitute about $6 \%$ of whole therapeutic agents used in clinics. These drugs microtubule organization and cause onset of apoptotic mechanisms in affected cells, leading to death of cells. Tubulin-binding drugs either prevent microtubule polymerization (Vinca alkaloids) or block their disassembly (Taxol alkaloids) [2], both leading to cell death. Due to less specificity of common tubulin-binding drugs, these agents bind to non-specific targets and cause side effects. Inception of resistance and availability of target in non-malignant cells also leads to non-specific toxicity to normal cells.

Drugs of Vinca and Taxane alkaloids are limited in use due to the number of reasons including non-specific toxicities to sensory neurons (neuropathies) and digestive tract (gastritis). These drugs also cause alopecia and cause wreck myelopoiesis, leading to reduced number (cytopenia) and function of cells of immune system. Perturbed cellular homeostasis caused by Taxol derivatives and other, many other antimitotic drugs affect expression of inflammatory mediators such as tumor necrosis factor-alpha and interleukins, and other enzymes including nitric oxide synthase and cyclooxygenase. Therefore, it becomes imperative to search and screen new drugs or prepare derivatives of existing microtubule-binding agents with high specificity, less toxicity to normal cells, and other side effects.

Noscapine and triazole have been reported for their MT-binding activity with less toxicity. Noscapine is a benzylisoquinoline from plants of the poppy family, without painkilling properties. It was discovered by French pharmacist and professor Pierre-jean Robiquet, in 1817, from opium plant (Papaver somniferum). This agent was primarily used for its antitussive (cough suppression) effects [3]. Nevertheless, later, it was found to have cytotoxic activity against cancer cells in vitro [4,5]. Due to less economic value during the $60 \mathrm{~s}$, noscapine was no further analyzed for its potential to treat cancer. However, in 1998 [4], anticancer potential of noscapine was revisited and analyzed by structure-based rationale screening of library of natural compounds sharing structural similarities with toxic MT depolymerizing drugs such as colchicines, podophyllotoxin, and MTC [2,3,4-trimethoxy-4-acetyl 1,1-biphenyl]. Mechanistic studies of noscapine suggest stoichiometric interaction 
with tubulin, leading to altered conformation causing arrest of cell division. Noscapine causes alteration in the steady-state dynamics of microtubule assembly before and during metaphase [6]

Triazole $\left(\mathrm{C}_{2} \mathrm{H}_{3} \mathrm{~N}_{3}\right)$ is a heterocyclic compound having a five-membered ring of two carbon atoms and three nitrogen atoms [7]. There are two sets of isomer, which differs in their relative positions of the three nitrogen atoms. The in vitro studies performed by National Cancer Institute (NCI), USA, using a panel of 60 human cancer cell lines (NCI60), have shown that 1,2,3 triazole and its derivative poses anticancer activity [7]. On an average, it takes about 10-15 years and US\$500800 million to introduce a drug in the market. The use of computeraided drug designing to improve the efficacy of the drug discovery and will accelerate the development pipeline in the pharmaceutical industry. Utilizing tools and applications of bioinformatics for in silico development and synthesis of novel drugs for the treatment of various diseases will improve the effectiveness with reduced cost and time.

Combinatorial chemistry of many derivatives of noscapine has been used along with reasonable speculative prediction model of most potent derivative (Figs 1 and 2). Despite being primary tool for speculative calculation, the use of molecular docking alone may result in decrease in accuracy. To overcome this issue and find more precise predictive information about derivatives, the present study employs methodologies to rescore docking results. The present investigation exercises molecular mechanics in combination with implicit continuum salvation-based scoring function including linear interaction energy method with a surface generalized born continuum salvation model, generalized born solvation area, etc., in calculating the binding affinity between protein and ligands. This scheme was used to find out accurate

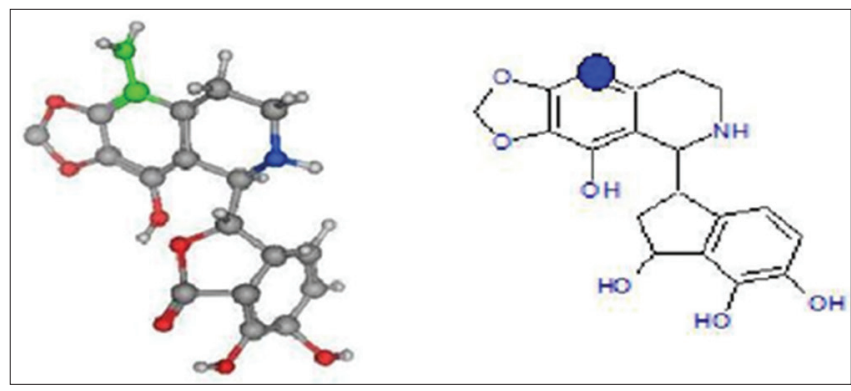

Fig. 1: Scaffold structure of Noscapine with predicted $\log P$ value of $0.11 \pm 0.68$, and $13^{\text {th }}$ carbon atom (marked as green) is the position where different triazole group is substituted

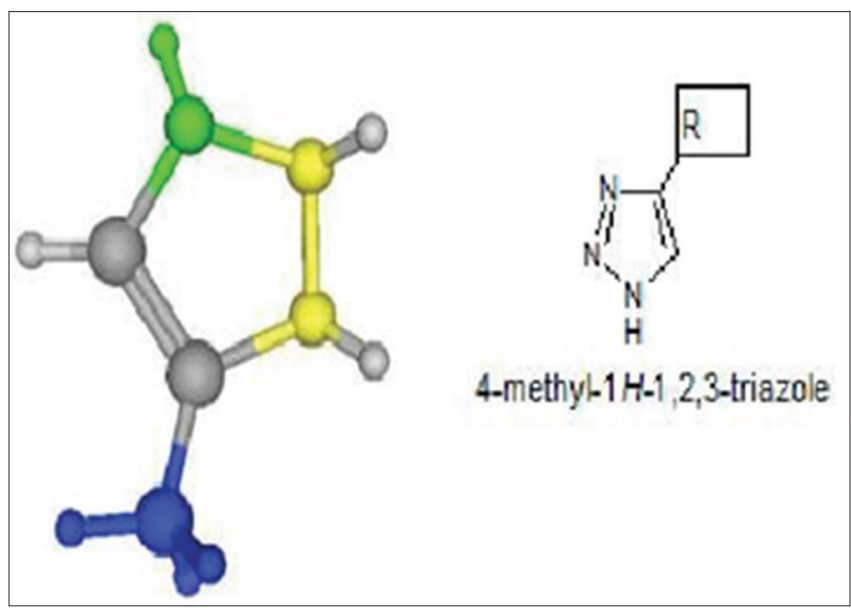

Fig. 2: Triazole molecule having different " $R$ " groups at $4^{\text {th }}$ carbon atom is substituted to the $13^{\text {th }}$ position of Noscapine. The marked green portion is Noscapine joining portion, whereas the blue marked is different functional group substitution exploratory information about generated derivatives from noscapine and triazole, with high efficacy and predictive clinical applicability.

\section{METHODS}

\section{In silico screening and ligand preparation}

\section{Selection of protein file}

The criteria for the selection of protein file (PDB) were that it should be from the human source and should have a resolution between 0.5 and 2.00 A. Thus, PDB file of ABTUBMDS was selected. The PDB was downloaded from RCSB protein data bank. The allowable and disallowable regions were checked from Ramachandran plot and ERRAT report of PDB. The water molecules were removed from the monomer. The hydrogens were added to the protein molecule and energy was minimized using Merck molecular force field (MMFF).

\section{Ligand preparation}

Ligands from the series of 3-(1H-1,2,3-triazole-4-yl)propan-1-ol derivative of noscapine (Table 1) to (3R,4S)-5-(1H-1,2,3-triazol-4-yl) tetrahydrofuran-2,3,4,triazole derivative of noscapine were prepared by the combinatorial approach in MOE Builder (MOE 2008.10). The 3D structure was energetically minimized using molecular mechanics followed by MMFF. All the conformers were then energetically minimized up to the RMS gradient of 0.001 and saved in a separate folder. Dielectric properties were kept constant. Gradient type used was analytical with a maximum number of cycles were 1000 . The potential energy of the conformers was calculated using MOE potential energy calculator.

\section{LogP value}

Log P stands for the partition coefficient of any compound; it measures how well a substance partitions between a lipid (oil) and water. It helps in understanding the behavior of drug molecules, as different ionic species of molecule differ in its physiochemical and biological property so it is important to be able to predict which ionic form of the molecule is present at the site of action.

$$
\begin{aligned}
& \log P=1 \text { means } 10: 1 \text { organic: aqueous } \\
& \log P=0 \text { means } 1: 1 \text { organic: aqueous } \\
& \log P=-1 \text { means } 1: 10 \text { organic: aqueous }
\end{aligned}
$$

Studies have found: (these may not apply to every class of chemicals)

- Optimum CNS penetration around Log $\mathrm{P}=2 \pm 0.7$ (Hansch)

- Optimum Oral absorption around $\log P=1.8$

- Optimum Intestinal absorption Log P =1.35

- Optimum Colonic absorption $\log P=1.32$

- Optimum Sublingual absorption Log P = 5.5

- Optimum Percutaneous Log $\mathrm{P}=2.6$ (and low $\mathrm{mw}$ ).

Formulation and dosing forms:

- Low Log P (below 0) Injectable

- Medium (0-3) Oral

- High (3-4) Transdermal

- Very High (4-7) Toxic buildup in fatty tissue.

\section{Lipinski's "rule of 5 " for drugs}

Chris Lipinski of Pfizer derived an easy to use "rule of thumb" for druglikeness in molecules after surveying the world's marketed drugs.

The rule states that for reasonable absorption:

- Keep H-Bond donors <5 (sum of $\mathrm{OH}$ and $\mathrm{NHs}$ )

- Keep $\mathrm{mW}<500$

- Log P should be $<5$

- No more than $10 \mathrm{H}$ bond acceptors.

\section{Energy minimization}

The energy minimize application in MOE calculates atomic coordinates that are local minima of a molecular energy function. Energy minimization comprises finding a set of atomic coordinates that match up to a local minimum of the molecular energy function (such as the 
Table 1: Different functional groups attached to triazole moiety and their LogP value mainly partitioned between water and octanol

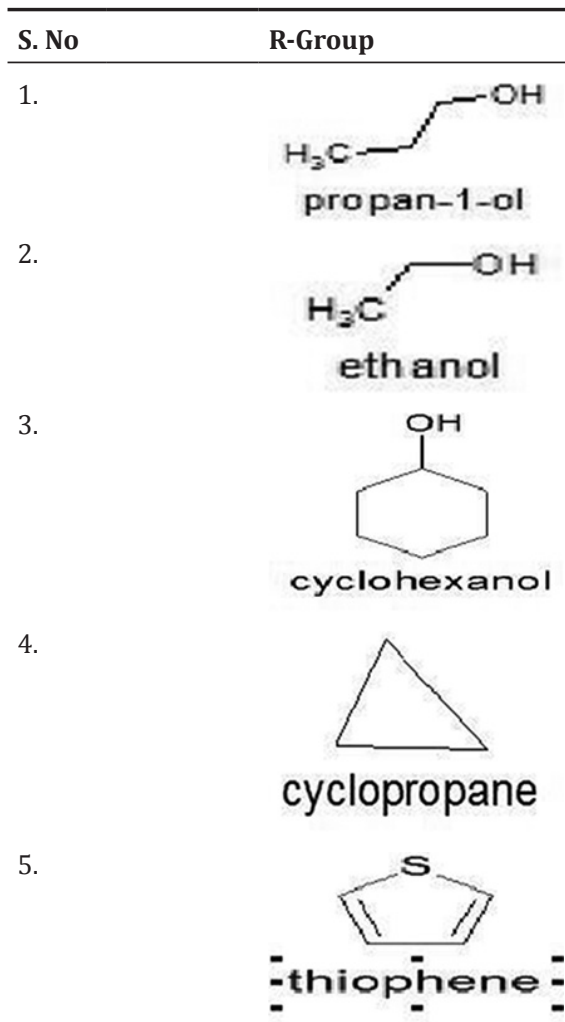

6.

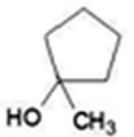

1-methylcyclopentanol

7.

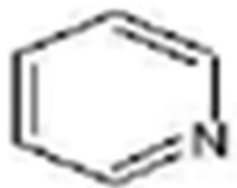

pyridine

8.

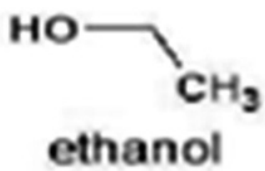

$0.19 \pm 0.18$

$2.82 \pm 0.93$

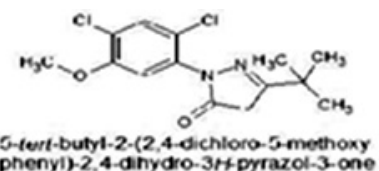

10.

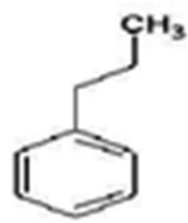

$3.74 \pm 0.17$

propylbenzene
20.

Table 1: (Continued)

\begin{tabular}{lll}
\hline S. No & R-Group & LogP \\
\hline 11. & & \\
& &
\end{tabular}

12.<smiles>Cc1ccccc1</smiles>

$2.68+0.17$

methylbenzene

13<smiles>CC(C)(C)c1ccccc1</smiles>

$3.19 \pm 0.19$

tert-butylbenzene

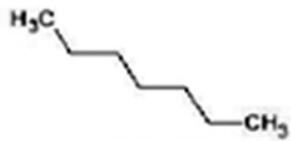

heptane

15.<smiles>Cc1ccccc1</smiles>

methrounzene

16.

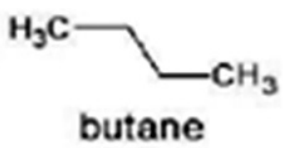

$2.88 \pm 0.16$

$3.98 \pm 0.23$

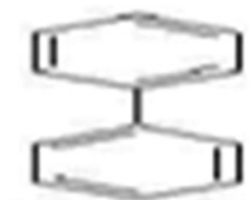

bisterey!

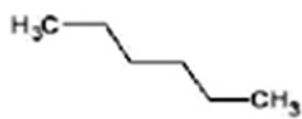

hexane

19.

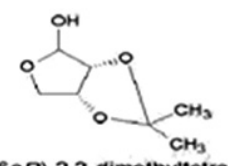

(3aR,6aR)-2,2-dimethyltetra hydrofuro[3,4-a][1,3]dioxol-4

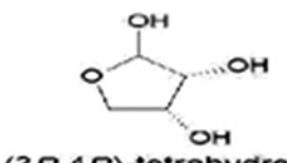

(3R,4R)-totrahydro furan-2,3,4-triol 


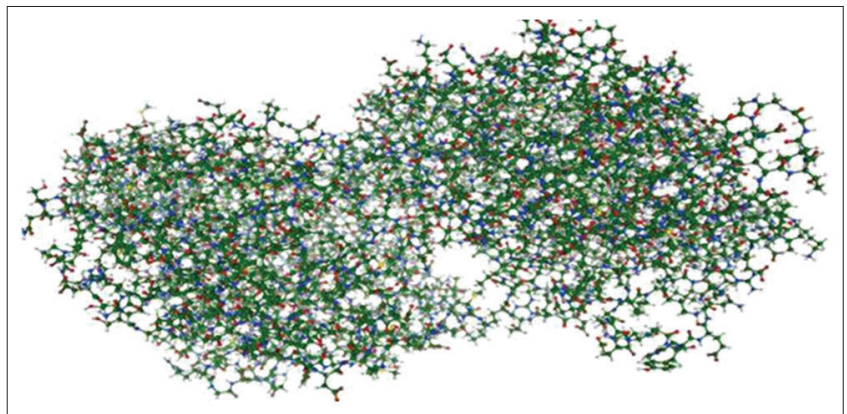

Fig. 3: ABTUB protein (Receptor molecule) to which hydrogen is added, partial charge is added, automated connection and type bonds were made, and potential energy is fixed

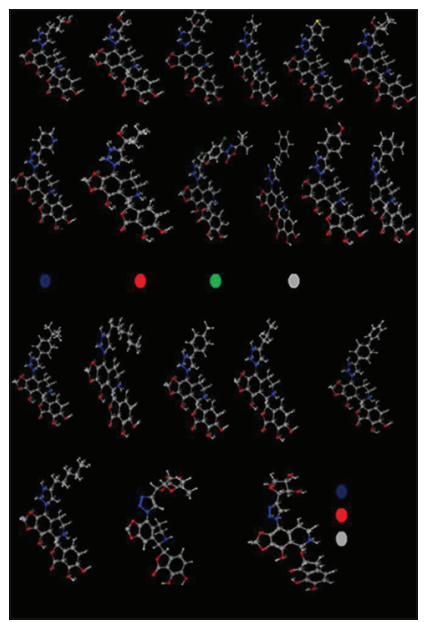

Fig. 4: Ligand prepared by combinatorial approach, resulting in fused pharmacophore derivative of noscapine and triazole

potential energy model). This is done by applying large-scale nonlinear optimization techniques to calculate a conformation (near to the starting geometry) for which the forces on the atoms are zero.

\section{Force field optimization}

The truncated Newton (TN) method is the most efficient large-scale non-linear optimization method known. It exhibits superlinear convergence even in highly non-linear conditions. The TN method attempts to use curvature information to improve convergence. The Newton direction $p$ satisfies:

$\operatorname{Gk}(\mathrm{xk}) \mathrm{p}=-\operatorname{grad} \mathrm{U}(\mathrm{xk})$

Where, Gk is the second derivative, or Hessian, of U. These equations are called the Newton equations. TN solves the Newton equations approximately using an iterative linear equation solver. The iterative linear equation solver (based on the Linear Conjugate Gradient method) is terminated after a small number of iterations, hence, the name TN. The energy of both receptor and ligand is minimized.

\section{Binding site}

The different site of ligand binding is detected using "site finder" in MOE (MOE 2008.10). The purpose of Site Finder is to calculate possible active sites in a receptor from the 3D atomic coordinates of the receptor. Site Finder falls into the category of geometric methods since no energy models are used. Instead, the relative positions and accessibility of the receptor atoms are considered along with a rough classification of chemical type. The Site Finder methodology is based on Alpha Shapes (AlphaShapes) which are a generalization of convex hulls developed in (Edelsbrunner, 1995). About 46 binding sites are detected to which binding of ligand is performed.

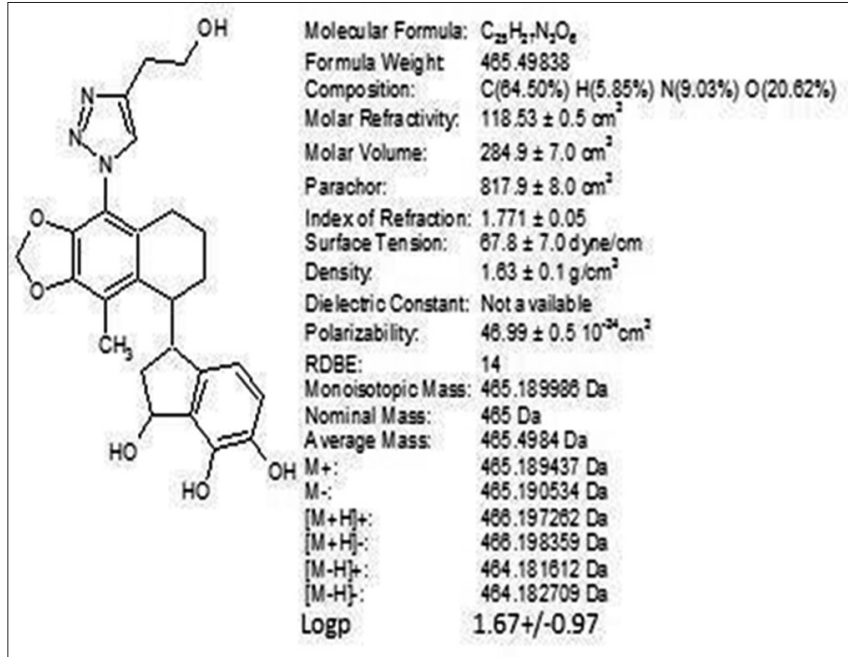

Fig. 5: Molecular property of ligand 1 that showed maximum efficacy

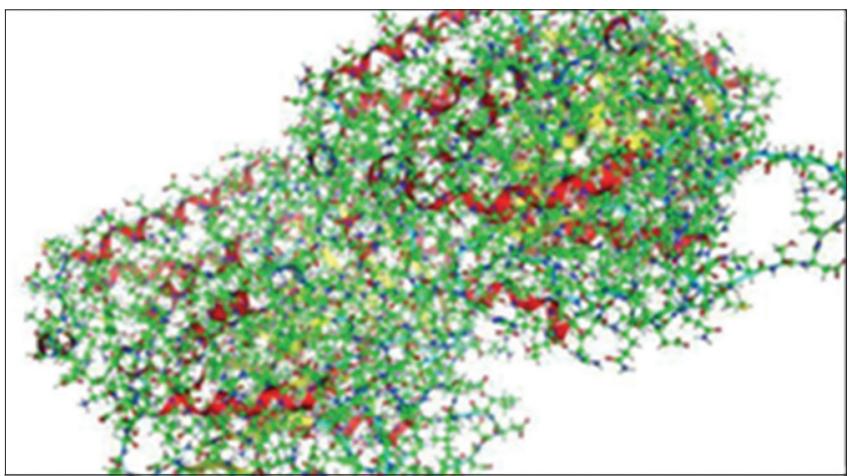

Fig. 6: Sites generated by Site Finder in ABTUB protein at alphaposition

\section{Surface and mapping}

The surfaces and maps application is used to generate visual representations of molecular surfaces and can be used to predict the preferred locations of ligand atoms (in the absence of the ligand itself). The surfaces and maps application is capable of calculating a number of molecular surfaces - surfaces near to the Van der Waals or solvent accessible surfaces of a molecule. The reentrant surface, or Connolly surface, consists of the boundary of the volume of space defined by removing all points inside the Van der Waals surface of the spherical probe (that represents the solvent) located at all positions for which the probe does not contact the Van der Waals volume of a molecule.

\section{Molecular docking}

The purpose of the dock application is to search for favorable binding configurations between small- and medium-sized ligands and a not too flexible macromolecular target, which is usually a protein. For each ligand, a number of configurations called poses are generated and scored in an effort to determine favorable binding modes. The top scoring poses are written out to a database for further analysis. Docking simulation was carried out using MOE-Dock. The default parameters of MOE-Dock program were used to find the correct conformations of the ligands and to obtain minimum energy structures. Ligands were allowed to be flexible (rotation of bonds was allowed) in the docking algorithm. At the end of docking, the best conformations of the ligands were analyzed for their binding interactions. The parameters used for docking were as follows: Triangle Matcher placement with timeout (300 s) and the number of Return poses $=1000$, Rescoring1: London $\mathrm{dG}$, Retain $=10$, and force field refinement with a final gradient of 0.01 with a maximum of 500 iterations. The docking of the $28 \mathrm{CCBs}$ into the 


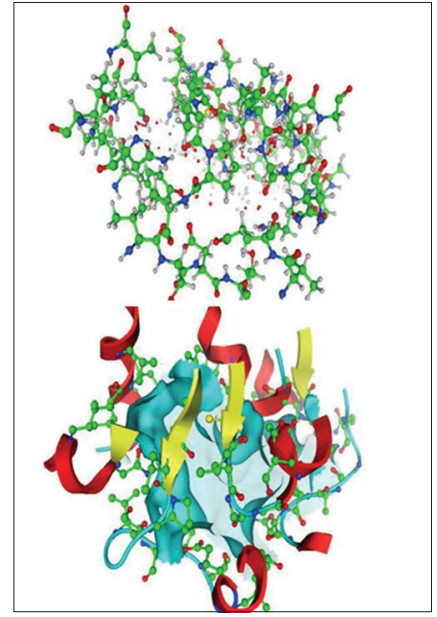

Fig. 7: Alpha dummies atom at site 2 of ABTUB receptor and molecular surface at that site

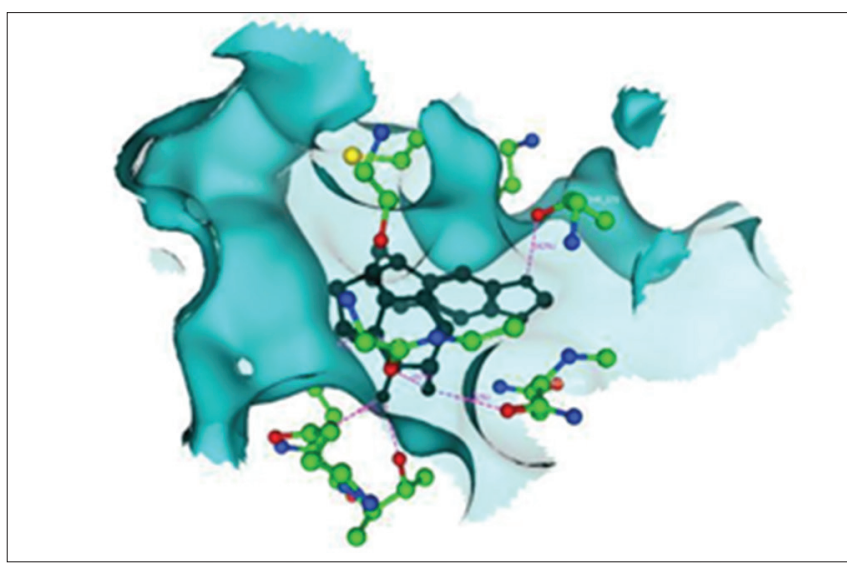

Fig. 8: Native Noscapine to site 2

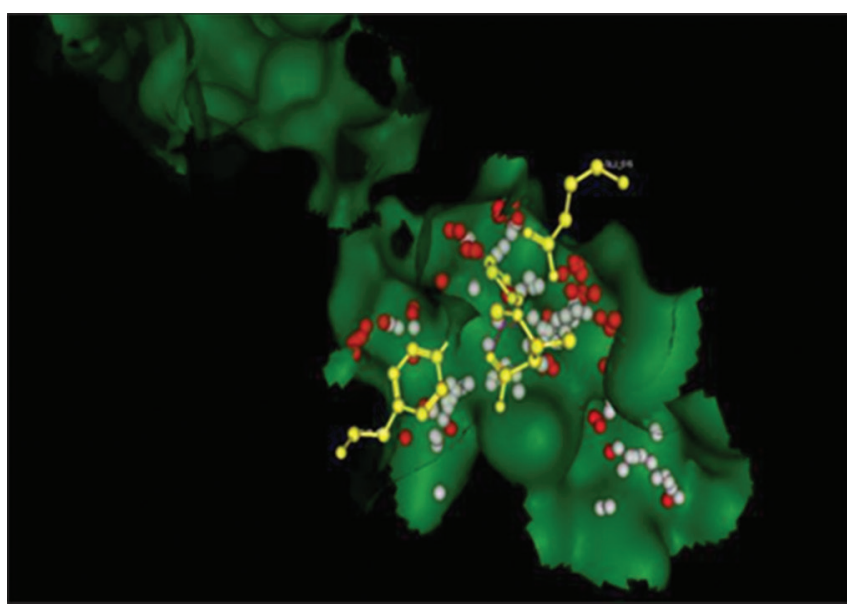

Fig. 9: Native Triazole to site 2

receptor was simulated. Each docking simulation was evaluated based on the EScore 1 value, which indicates the interaction energy between the ligand and the ABTUB ligand-binding site.

\section{Ligand interaction}

The ligand interactions application provides a means to visualize an active site of a complex in diagrammatic form. The diagram itself consists of the selected ligand as the centerpiece, which is drawn using the traditional schematic style for molecules. A selection of interacting entities, which includes hydrogen-bonded residues, close but non-bonded residues, solvent molecules, and ions are drawn about the ligand, their positions in $2 \mathrm{D}$ being chosen to be representative of the observed 3D distances, as well as taking into account esthetic considerations. Additional properties such as solvent-accessible surface area and the ligand proximity outline are also shown.

\section{LigX energy calculation}

LigX is a tool of MOE, which is used to explore protein-ligand interaction, and help in manual constructing of the novel compound in protein binding sites, and evaluate for binding free energy and affinity calculation. In LigX minimization, the receptor atoms near ligand (active site) are allowed to move while the receptor atoms far from the ligand are held fixed (constrain not to move) but are a subject of tether restraints that discourage gross movement. The ligand atom can either be free to move or subject to similar tethers specified by a user. When we run a tethered energy minimization while the ligand properties are displayed, the MM/GBVI binding free energy, affinity, and efficiency will be calculated and added to the ligand properties.

\section{RESULTS}

\section{Selection of protein file}

ABTUB protein file is downloaded from the public database library in the PDB format and is then exported to the MOE window where partial charges and hydrogen is added, and the potential energy of the receptor is fixed using MOE/EDIT function tool (Fig 3).

\section{Ligand preparation}

Using combinatorial approach, 20 derivatives of ligand are made. Using Molecular Builder tool (MOE 2008.10), ligand is made by adding different functional group containing triazole pharmacophore to the biaryl pharmacophore skeleton of noscapine (Fig 4). Mainly addition of triazole is made at the $13^{\text {th }}$ carbon position in the skeleton of noscapine. On the basis of it, about 20 stable structures were drawn.

The energy of all the 20 ligands is minimized and saved in MDB file format. However, the ligand 1 [2-(3H-pyrazol-5-yl)ethanol ammoniate (1:1) derivative of noscapine] showed maximum activity in overall process; hence, the overall property of the ligand (Fig 5) is deduced using software ChemDraw, the property generated is as follows:

\section{Site generation}

Using Site Finder tool in MOE/compute/site finder, sites are generated at alpha center and dummy atoms are created. All the total of 46 binding sites was generated (Fig 6) to which there is a possibility of ligand binding. A list of site is generated to which each and every site is saved in MOE format for further use.

\section{Surface and map generation}

A Gaussian surface is generated (Figs7-9) at alpha dummies atom surrounding the Van der Waals surface of a molecule which is solvent in accessible gaps with constant activity, colored by constant properties and the transparency of back side is set to more than $75 \%$ approximately. Using graphics, object manager back and front is made glossy.

\section{Molecular docking}

Docking is performed for every ligand to each site and is searched for maximum binding energy score value (Figs10 and 11). However, the binding energy value of ligand 1 with that of site 2 showed a maximum result. Docking is also performed with native triazole and noscapine molecule to the same site.

The overall binding score was -16.8035 , with retain of 10 , then the same was exceeded to retain a value of 100 , leading to more optimized result, i.e., -16.0380 . While the overall binding of native noscapine to the same site is -12.0377 , and that of native triazole molecule is -6.7858 . Hence, the binding efficacy of the new fused molecule of both the native compounds has increased. 


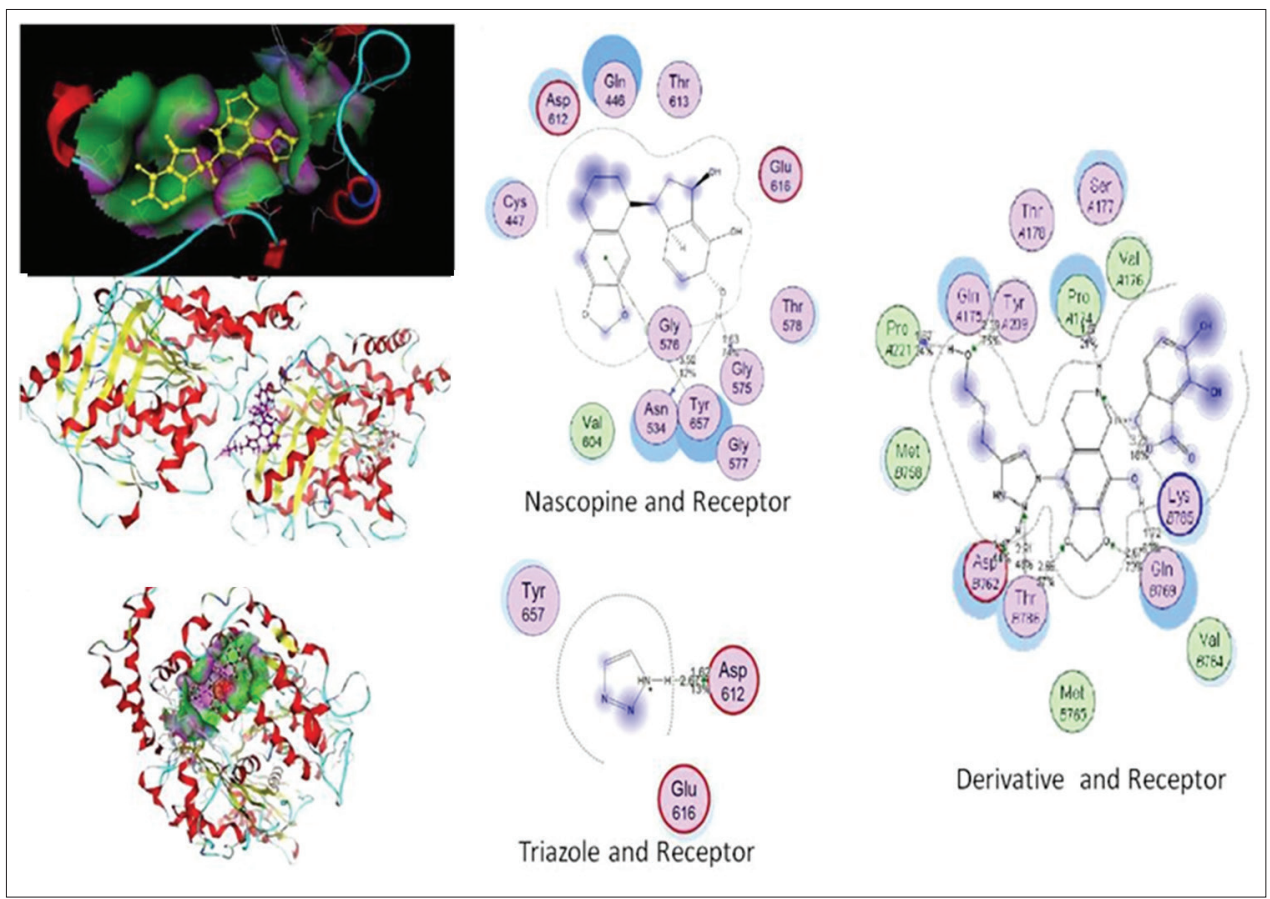

Fig. 10: Docking image between ligand 1 [2-(3H-pyrazol-5-yl)ethanol ammoniate(1:1) derivative of Noscapine] and Receptor[Alpha-Beta Tubulin]

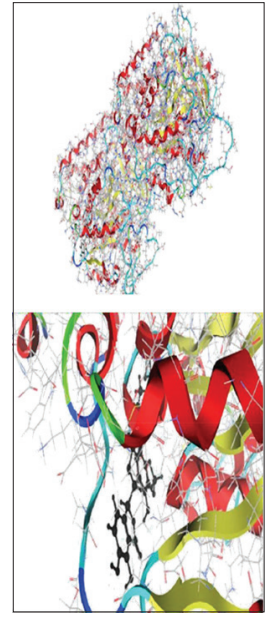

Fig. 11: Simulated snapshot of ligand-protein complex. Where black color molecule is Ligand

\section{Ligand interaction}

With the help of LigX plot, interaction plot is generated which provides a detailed knowledge of the type of bonds and amino acids playing a role in the interaction. The interaction report and diagram of triazole and noscapine and then the ligand is attached one by one. The interacting amino acid in case of native triazole is aspartic acid and in case of noscapine is glycine, asparagine, and tyrosine; whereas, in case of my ligand 1 [2-(3H-pyrazol-5-yl)ethanol ammoniate (1:1) derivative of noscapine] has lysine, glutamine, threonine, asparagine, proline, and tyrosine; hence, we can say that the overall binding efficiency of the new fused compound has increased from its native molecule (Fig 12).

\section{MD simulation}

MD simulation is carried out for 200 picosec in two phases, i.e., neutral and heating phase. The value calculated out after equilibrium simulation is 0.001 and the correlation plot is shown below. The basic principle behind MD simulation is that every particle has its own vibrational motion and frequency which changes from one state to other state and then to a zeroth stage. Thus, MD simulation helps in identifying the stability of substance at two-point stage and then time back to its zeroth stage and the kinetic energy of motion is deduced.

\section{DISCUSSION}

The side chain addition is done keeping in mind their partition coefficient. The ligand derivative generated was force field energy optimized, and the activity was deduced by calculating the binding energy and free energy binding (MM/GBVI) of ligand and native compound. The result generated has shown an elevated result of drug efficacy from its lead compound, i.e., noscapine and triazole. Native binding energy generated by docking is in the range of 4 for triazole and 11 in the case of noscapine while to an astonishing my lead compound showed binding energy in the range of 16 , which is much more efficient. Similar to that, the MM/GBVI energy of triazole cannot be estimated as it is a small molecule with high vibrational energy, while that of noscapine showed an energy of $-16.272 \mathrm{kcal} /$ $\mathrm{mol}$ and that of my ligand has $-23.208 \mathrm{kcal} / \mathrm{mol}$ not only this ligand interaction showed an increase in binding amino groups but also the percent of binding was $13 \%$ with aspartic acid for triazole, 12\% with tyrosine, and $74 \%$ with glycine for noscapine. While the ligand has $75 \%$ with tyrosine, $24 \%$ and $21 \%$ proline at two sites, $44 \%$ with aspartic acid, $48 \%$ and $57 \%$ with threonine from two sites, $73 \%$ and $63 \%$ with glutamic acid at two sites, and $18 \%$ with lysin, this shows that the overall binding affinity has increased and thus the drug can be screened for in vivo and ex vivo experiment to support the in silico result, and finally, it can be used as one of the oral drugs of cancer in future as the $\log \mathrm{P}$ result shows it to be non-toxic and oral route administration is predicted.

\section{CONCLUSION}

In the present study a greater affinity of the newly designed ligands for binding to tubulin was determined for 2-(3H-pyrazol-5-yl) ethanol ammoniate (1:1) derivative of noscapine. The predictive binding free energy (Gbind,pred) of all twenty derivatives were ranging from -10.5178 to $-16.8473 \mathrm{kcal} / \mathrm{mol}$ based on linear interaction energy 


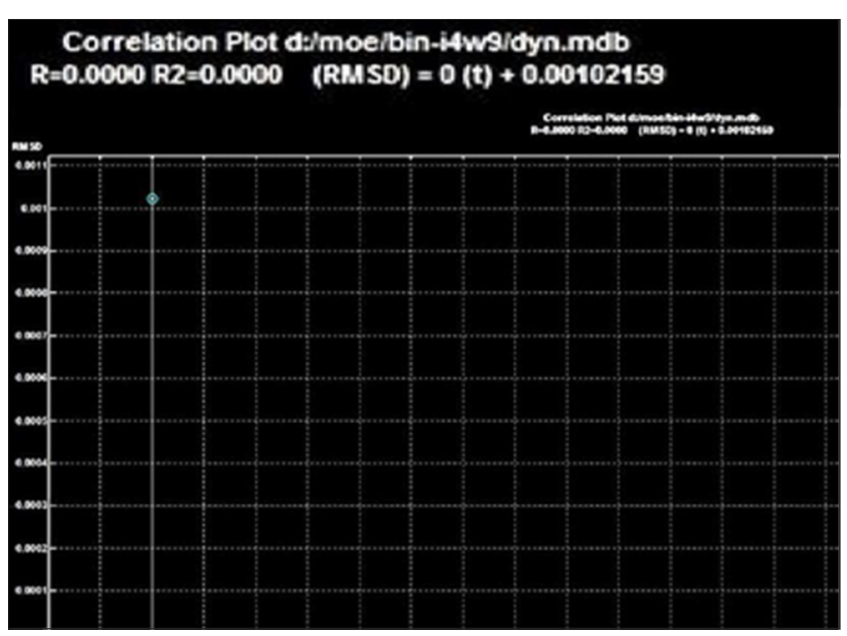

Fig. 12: RMSD plot of LIGAND-RECEPTOR complex

method with a surface generalized born continuum salvation model which shows improved binding affinity with tubulin as compared to the lead compound. Natural $\alpha$-Noscapine $(-5.505 \mathrm{kcal} / \mathrm{mol})$. Therefore the derivatives can be further evaluated for its anticancer activity by using invitro and invivo techniques.

\section{REFERENCES}

1. Fife CM, McCarroll JA, Kavallaris M. Movers and shakers: Cell cytoskeleton in cancer metastasis. Br J Pharmacol 2014;171:5507-23.

2. van Vuuren RJ, Visagie MH, Theron AE, Joubert AM. Antimitotic drugs in the treatment of cancer. Cancer Chemother Pharmacol 2015;76:1101-12.

3. Idanpann-Heikkila JE, Jalonen K, Vartiainen A. Evaluation of the antitrussive effect of noscapine and codeine on citric acid cough in guines-pigs. Acta Pharmacol Toxicol 1967;25:333-8.

4. Eagle H, Foley G. Cytotoxicity in human cell cultures as a primary screen for the detection of anti-tumor agents. Cancer Res $1958 ; 18: 1017-25$.

5. Lettre H. Synergists and antagonists of mitotic poisons. Ann NY Acad Sci 1954;58:1264-75.

6. Ye K, Ke Y, Keshava N, Shanks J, Kapp JA, Tekmal RR, et al. Opium alkaloid noscapine is an antitumor agent that arrests metaphase and induces apoptosis in dividing cells. Proc Natl Acad Sci USA 1998:95:1601-6.

7. El-Sayed WA, Flefel EM, Morsy E. Anticancer and antimicrobial activities of some synthesized pyrazole and triazole derivatives. Der Pharm Chem 2012;4:23-32. 